results from the removal of an interleukin-10producing regulatory $\mathrm{B}$-cell subset, termed B10 cells, before EAE initiation. By contrast, the beneficial effect of treatment on day 14 is probably attributable to reductions in $\mathrm{CD}^{+}$and pathogenic T-cell proliferation.

The development and use of B-cell depletion therapies should take into account, therefore, the timing and nature of B cell effects on disease initiation and pathogenesis.

Original article Matsushita T et al. (2008) Regulatory B cells inhibit EAE initiation while other B cells promote disease progression. J Clin Invest 118: 3420-3430

\section{Bone morphogenetic protein 7 inhibits cartilage degradation in a rabbit model of osteoarthritis}

A well-designed study suggests that intraarticular injections of bone morphogenetic protein 7 (BMP7) prevents the progression of cartilage damage in a rabbit anterior cruciate ligament transection model of knee osteoarthritis. In the study by Hayashi and colleagues, 12 weekly injections of $50 \mathrm{ng}, 500 \mathrm{ng}$ or 5,000 ng of recombinant human BMP7, or saline (control), were administered to 36 knees of 18 rabbits. Morphological and histological examination revealed that the two highest doses of BMP7 inhibited the progression of osteoarthritis to a similar extent. No evidence of increased synovial fibrosis, formation of ectopic bone or cartilage, or increased osteophyte size was detected in BMP7-treated knees. Furthermore, BMP7 concentrations in the cartilage of treated knees were still measurable by enzyme-linked immunosorbent assay 7 days after the last injection.

The authors addressed the issue of individual variability in osteoarthritis progression by use of a matched-pair analysis, whereby weekly $500 \mathrm{ng}$ injections of BMP7 in the right knees of 15 rabbits were compared with saline injected into the left knees of the same animals. At 12 weeks, knees injected with BMP7 had fewer cartilage erosions, which were limited to either the medial or the lateral condyle, than control knees, in which severe lesions were present in both locations. In addition, histological scores were better in treated knees compared to the contralateral control knees.

Intra-articular injection of BMP7, therefore, has potential as a nonsurgical alternative for inhibiting cartilage degradation in osteoporosis. Future in vivo studies should investigate the optimal duration of this approach for osteoporosis of the knee.

Original article Hayashi M et al. (2008) Weekly intra-articular injections of bone morphogenetic-7 protein inhibits osteoarthritis progression. Arth Res Ther 10: R118

\section{Three genetic loci associated with risk for gout have been identified}

Via the use of genome-wide association studies, Dehghan et al. have confirmed a previously identified association between genetic variation in SLC2A9 and both serum uric acid concentration and risk of gout in white individuals. In addition, they demonstrated that this same association was present in black people, and identified two new candidate loci (in ABCG2 and SLC17A2) that show similar associations.

The initial genome-wide screen looked for gene variants that correlated significantly with levels of uric acid in participants from the Framingham and Rotterdam cohorts. Five candidate single-nucleotide polymorphisms were identified, clustered within three genetic loci. The Atherosclerosis Risk in Communities (ARIC) study genotyped three of these polymorphisms and found associations between them (one per locus) and both serum uric acid concentration and risk of gout. All three loci are thought to influence renal urate transportation. The number of risk alleles harbored by individuals in all three cohorts showed strong, linear correlations with serum uric acid concentrations and with prevalence gout. Across all three cohorts, the prevalence of gout in individuals without risk alleles was 1-2\%, which rose to $8-18 \%$ in those with six risk alleles.

The number of risk alleles present in an individual could potentially be developed as a screening tool to predict the risk of gout in asymptomatic individuals, to decide whether asymptomatic hyperuricemia should be treated, and to ensure that individuals with a putative genetic predisposition to gout do not receive treatments contraindicated in that condition.

Original article Dehghan A et al. (2008) Association of three genetic loci with uric acid concentration and risk of gout: a genome-wide association study. Lancet [doi:10.1016/ s0140-6736(08)61343-4] 